\title{
AVALIAÇÃO COMPORTAMENTAL DE CRIANÇAS PRÉ-ESCOLARES EM PROGRAMA DE NATAÇÃO
}

\section{ARTIGO ORIGINAL}

FERREIRA, Maria Fernanda Lopes ${ }^{1}$

NAGAMINE, Kazuo Kawano ${ }^{2}$

FERREIRA, Maria Fernanda Lopes. NAGAMINE, Kazuo Kawano. Avaliação comportamental de crianças pré-escolares em programa de natação. Revista Científica Multidisciplinar Núcleo do Conhecimento. Ano 04, Ed. 10, Vol. 08, pp. 7790. Outubro de 2019. ISSN: 2448-0959, Link de acesso: https://www.nucleodoconhecimento.com.br/educacao-fisica/avaliacaocomportamental

\section{RESUMO}

O comportamento esportivo é crucial durante a infância e adolescência, pois o crescimento e o desenvolvimento neurofuncional são acelerados. Avaliação do comportamento infantil é importante para identificar dificuldades e auxiliar nos diagnósticos comportamentais e intervenções. O objetivo deste estudo foi avaliar o desenvolvimento comportamental de crianças pré-escolares em um programa de natação. Participaram 45 crianças entre 3 e 5 anos, matriculados em uma escola de natação, na cidade de São José do Rio Preto. Foi aplicado o questionário Child Behavior Check List CBCL / 1 e 5 anos, antes e depois do programa de natação. Para análise estatística utilizou-se: Qui-quadrado de McNemar, Prisma, e o Teste de

\footnotetext{
${ }^{1}$ Doutorado em andamento em Biofísica Molecular; Mestrado em Psicologia e Saúde; Graduação em Educação Física.

${ }^{2}$ Doutorado em Ciências da Saúde, Aperfeiçoamento em Fisioterapia. Graduação em Educação Física.
} 
Wilcoxon. Descobriu-se redução dos casos clínicos e melhora significativa em seis síndromes após o programa.

Palavras-chave: Natação, crianças, comportamento, desenvolvimento.

\section{INTRODUÇÃO}

Borsa \& Nunes (2011) apontam que há prevalência 12,5\% de transtornos mentais na população infanto-juvenil no Brasil e no mundo, e identificam os problemas de comportamento como um dos mais prevalentes em crianças e adolescentes, Borsa \& Nunes (2011). Na Holanda, aproximadamente 10 a 25\% das crianças pré-escolares têm comportamento problema ou dificuldades emocionais e somente uma, em cada cinco crianças, recebe cuidados psicológicos (THEUNISSEN, VOGELS, WOLFF, CRONE \& REIJNEVELD, 2015).

Nos países de primeiro mundo, a população adulta tem filhos em idade mais tardia, e associa-se a gravidez em idade avançada com o aumento de doenças mentais e disfunções comportamentais, com um maior risco, principalmente de depressão, em crianças que têm pais tanto muito jovens quanto mais velhos (TEARNE, ROBISON, JACOBY, NEWNHAM \& MCLEAN, 2015). Os comportamentos problema e dificuldades emocionais tendem a iniciar-se na idade pré-escolar e persistem ao longo do desenvolvimento, causando prejuízos em muitas áreas do funcionamento infantil (PSYCHOGIOU, MOBERLY, PARRY, NATH, KALLITSOGLOU \& RUSSELL, 2017). Os comportamentos problema são classificados em internalizantes (retraimento, depressão, ansiedade e queixas somáticas) e externalizantes (impulsividade, agressão, agitação, características desafiantes e antissociais) (BOLSONI-SILVA, LOUREIRO \& MATURANO, 2011).

A relação entre pais e filhos no início da vida está associada a uma série de ações, por meio das quais o indivíduo inicia e mantém relações afetivas estáveis (ROHENKOHL \& CASTRO, 2012). O relacionamento dos pais é apontado como fator importante para a qualidade de vida das famílias, influenciando nos cuidados com os 
filhos e nas relações entre pais e filhos. O apoio dos às mães é um determinante para o desenvolvimento saudável dos filhos.

A avaliação e análise do comportamento de crianças são importantes e necessárias para identificar as dificuldades e viabilizar recursos para auxiliar nos diagnósticos comportamentais e, subsequentes intervenções, seja com as próprias crianças e/ou com seus pais/cuidadores (BOLSONI-SILVA, et al., 2011).

O aumento crescente de problemas de saúde mental tem motivado pesquisadores do mundo todo a estudar fatores que forneçam proteção ou que reduzam a vulnerabilidade para o desenvolvimento de transtornos mentais. Além do mais, há necessidade de encontrar métodos que sejam mais eficientes do que os tratamentos existentes, bem como adotar uma postura preventiva (BENKO, FARIAS \& CORDEIRO, 2011).

O comportamento esportivo tem valor singular durante a infância e adolescência, quando o crescimento e o desenvolvimento neurofuncional ocorrem de maneira mais acelerada e disfunções nutricionais podem prejudicar o funcionamento físico e mental dessas crianças. Assim, atenção especial à prática monitorada de exercícios físicos pode ser de vital importância ao desenvolvimento adequado dos processos neurobiológicos durante este período, ajudando a combater a obesidade e suas comorbidades, contribuindo, assim, para melhor saúde física e mental das crianças (BENKO, et al., 2011).

Os mecanismos sociais e comportamentais, por meio dos quais a prática esportiva afeta a saúde, são identificados em relação aos determinantes do comportamento saudável, o esporte pode melhorar a saúde devido às influências positivas nas habilidades de sociabilização encorajadas pelos professores, instrutores, líderes e, também, devido às exigências disciplinares de se fazer qualquer atividade em grupo (JONES-PALM, \& PALM, 2015). O esporte favorece a resiliência pessoal e a autoestima, promovendo uma oportunidade de aprender atitudes sociais saudáveis, pode melhorar a autoconfiança, fornece a sensação de capacidade física, além de 
possibilitar o reconhecimento social dentro da escola, em casa e na sociedade. (JONES-PALM, et al., 2015).

Dentre os esportes que atendem ao objetivo de melhorar o desenvolvimento de crianças na primeira infância, a professora Robyn Jorgens, da Universidade de Griffith, na Austrália (2012), diz que a natação é o esporte mais adequado, por possibilitar a prática de crianças bastante pequenas, antes mesmo de aprenderem a andar, pois bebês de 4 meses já podem começar atividades aquáticas. Jorgens (2012) constatou que crianças menores de 5 anos que têm aula de natação conseguiram melhorar sua cognição (desenvolvimento intelectual) e seu desenvolvimento físico, bem como suas habilidades sociais e autoconfiança, de forma significativamente mais acelerada e consistente do que crianças da mesma idade não praticantes de natação (SELKE, 2014).

A Jorgens (2012) verificou que crianças que começam a aprender a nadar aos 4 ou 5 anos têm dificuldades na aprendizagem do esporte jamais enfrentadas por aquelas crianças que começaram a nadar quando bebês. Além disso, os escores básicos dos quesitos desenvolvimentais das crianças que nadam desde bebês são significativamente melhores do que os daquelas que começam a nadar mais velhas.

Pesquisas documentam que o efeito estimulante de programas de natação para crianças pequenas tem o potencial para aumentar a inteligência, a concentração, o estado de alerta e as habilidades de percepção, além de melhorar, consideravelmente, o desenvolvimento social, emocional e físico. Conferem ainda às crianças um estado de relaxamento e calma bastante desejável e benéfico à aprendizagem (GRIFFITH UNIVERSITY, 2012; WEST, 2012).

\section{JUSTIFICATIVA}

Portanto, alternativas terapêuticas mais generalizadas que visam a melhoria da qualidade de vida são mais seguras e benéficas para todos os portadores de comportamento problema e/ou transtornos mentais. Assim, atividades programadas como aulas de natação, oficina de artes, teatro, atletismo, dentre outras, mostram-se 
mais abrangentes e adequadas, principalmente, para crianças (KRIEGER, et al. 2013).

Do ponto de vista cientifico, o estudo de informações referentes ao desenvolvimento de crianças e a comportamentos infantis coletivos promove a possibilidade de se melhorar a saúde coletiva, bem como a qualidade dos serviços públicos e privados prestados à população infantil.

Outro aspecto relevante da pesquisa é que estudos de prevalência em saúde mental em idade precoce (os primeiros cinco anos de vida) mostram-se insuficientes e escassos. Tendo em vista que evidências neurobiológicas, econômicas, psicológicas e sociais demonstram que intervenções durante os primeiros anos de vida são altamente eficientes para reduzir os problemas psicossociais, é relevante obter dados nacionais sobre os problemas afetivos e de condutas na primeira infância e idade préescolar (LECANNELIER, EWERT, GROISSMAN, GALLARDO, BARDET, BASCUÑAN, \& RODRÍGUEZ 2014).

\section{OBJETIVO}

Este estudo propõe avaliar o comportamento de crianças de 3 a 5 anos em um programa de natação.

\section{MÉTODO}

\subsection{PARTICIPANTES}

Participaram 45 crianças de 3 a 5 anos, regularmente matriculadas em uma escola de natação de São José do Rio Preto, sendo 60\% meninos e 40\% meninas.

\subsection{MATERIAIS E INSTRUMENTOS}

Inventário de comportamentos de crianças entre 1/2 - 5 anos Child Behavior Checkilist (CBCL 1 1 1/2 - 5) (ACHENBACH \& RESCOLA, 2000), composto por 100 itens que levam 
a duas escalas resumidas: a escala de problemas internalizantes (emocional reativo, ansiedade/depressão, reclamação somática e isolamento) e a escala de problemas externalizantes (problemas de atenção e comportamento agressivo). O inventário também apresenta síndromes orientadas pelo Manual de Diagnóstico e Estatística de Transtornos Mentais (DSM), sendo elas: problemas afetivos, problemas de ansiedade, problemas desenvolvimentais, problemas de déficit de atenção e hiperatividade e problemas de comportamento opositivo desafiador. Este inventário fornece pontuações obtidas a partir de avaliação padronizada para crianças. A CBCL tem alta confiabilidade de teste e reteste e alta consistência interna. A validade relatada dos critérios de ambas as versões da CBCL é apoiada pela habilidade de suas pontuações quantitativas em discriminar entre crianças demograficamente pareadas, classificadas em casos clínicos e não clínicos (CIMINO, CEMIGLIA, PORRECA, SIMONELLI, RONCONI \& BALLAROTTO, 2016).

Questionário sociodemográfico, criado pela própria pesquisadora para identificar o perfil dos participantes, das crianças e dos responsáveis. O instrumento tem dezoito questões, sendo elas sobre a idade, sexo, cor da pele, naturalidade, tempo residindo na cidade, estado civil dos pais, escolaridade e situação ocupacional dos pais, número de pessoas que residem na casa, número de filhos, número de cômodos na casa e renda familiar.

Observações dos comportamentos das crianças feita pela professora, tabela elaborada pela pesquisadora para anotar, diariamente, os quesitos comportamentais das crianças ao longo do programa. Tal instrumento foi idealizado com o intuito de fornecer dados para possibilitar uma avaliação quantitativa dos comportamentos individuais. Cada criança teve um diário, com anotações dos comportamentos específicos apresentados em todas as aulas. Os quesitos avaliados foram: nível de atenção e concentração, agressividade, impulsividade, compulsão verbal, comportamento depressivo, capacidade de seguir comando e sociabilidade; foi considerada uma escala de 0 a 3, com 0 representando um comportamento menos adequado e 3, um comportamento mais adequado. Os quesitos comportamentais utilizados foram aqueles sugeridos por autores da literatura pesquisada neste trabalho 
(BOLSONI-SILVA, et al., 2011; JONES-PALM, et al., 2015; \& ROHENKOHL \& CASTRO, 2012).

Programa de natação, inclui rotinas e sequências de atividades compatíveis à faixa etária do grupo. As atividades foram dentro e nas imediações de uma piscina aquecida e coberta, de uma escola de natação de São José do Rio preto. Os exercícios foram sistemáticos e variados, visando estimular a consciência corporal, como o equilíbrio, a forca, a coordenação motora, a lateralidade, a noção de espaço, dentre outros.

Todas as atividades propostas são parte do programa didático e do conteúdo de ensino da escola em questão, cujo o objetivo é desenvolver, nas crianças dessa faixa etária, independentemente da experiência de natação, além da consciência corporal, a habilidade de deslocamento na água de 8 metros, tanto no estilo Cachorrinho (nado de sobrevivência), como no estilo Crawl rústico, e de um deslocamento de, pelo menos, 5 metros no estilo Costas.

As atividades foram cronometradas com duração variável, respeitando o limite físico e de tolerância de cada grupo. As sessões foram realizadas duas vezes por semana, com duração de 30 minutos. O programa foi desenvolvido em seis meses.

\subsection{PROCEDIMENTOS}

O estudo foi aprovado pelo Comitê de Ética em Pesquisa da FAMERP (Faculdade de Medicina de São José do Rio Preto/SP) - Parecer n.ำ1.420.285, no dia 23 de fevereiro de 2016.

O responsável de cada participante recebeu uma pasta com os questionários a serem preenchidos em julho de 2016. O mesmo recebeu novamente a pasta no mês de dezembro de 2016, apenas contendo o questionário CBCL para um segundo preenchimento. Assim, o CBCL foi aplicado em dois momentos, antes do programa de natação e após seis meses, com o objetivo de comparar os comportamentos das crianças 
Os dados obtidos pelo CBCL, foram analisados por meio de análise descritiva da pontuação T e contagem de frequência, para a classificação de clínico e não clínico. Também foi feita estatística inferencial utilizando o teste de Qui-quadrado de McNemar, utilizando-se os programas estatísticos Statistical Package For Social Sciences (SPSS versão 24.0) e prisma (versão 6.1, 2012). Os dados dos demais questionários foram analisados pelo Teste de Wilcoxon. Um nível de significância de $p \leq 0,05$ foi adotado em todas as análises.

\section{RESULTADOS}

A Tabela 1 mostra a comparação e a porcentagem dos casos clínicos de cada síndrome, para cada gênero, separadamente, para o número total da amostra e o valor de $\mathrm{P}$, antes e depois do programa de natação.

Tabela 1-comparação dos casos clínicos de cada síndrome, pré e pós programa de natação

\begin{tabular}{|c|c|c|c|c|c|c|c|c|c|}
\hline & \multicolumn{2}{|c|}{ MENINOS } & \multirow[b]{2}{*}{$\mathbf{P}$} & \multicolumn{3}{|c|}{ MENINAS } & \multicolumn{3}{|c|}{ AMOSTRA TOTAL } \\
\hline & $\begin{array}{l}\text { PRÉ } \\
\%\end{array}$ & $\begin{array}{l}\text { PÓS } \\
\%\end{array}$ & & $\begin{array}{l}\text { PRÉ } \\
\%\end{array}$ & $\begin{array}{l}\text { PÓs } \\
\%\end{array}$ & $\mathbf{P}$ & $\begin{array}{l}\text { PRÉ } \\
\%\end{array}$ & $\begin{array}{l}\text { PÓS } \\
\%\end{array}$ & $\mathbf{P}$ \\
\hline Emocional Reativa & 40,3 & 37,1 & 1 & 50 & 33,3 & $\begin{array}{l}0,4 \\
5\end{array}$ & 44,4 & 35,6 & $\begin{array}{l}0,3 \\
8\end{array}$ \\
\hline $\begin{array}{l}\text { Ansiedade/Depres } \\
\text { são }\end{array}$ & 22,2 & 18,6 & 1 & 27,8 & 16,7 & $\begin{array}{l}0,6 \\
2\end{array}$ & 24,4 & 17,8 & 0,5 \\
\hline $\begin{array}{l}\text { Queixas } \\
\text { Somáticas }\end{array}$ & 29,7 & 25,9 & 1 & 50 & 44,4 & 1 & 37,8 & 33,3 & $\begin{array}{l}0,7 \\
9\end{array}$ \\
\hline Isolamento & 37,1 & 25,9 & $\begin{array}{l}0,4 \\
5\end{array}$ & 50 & 33,3 & $\begin{array}{l}0,4 \\
5\end{array}$ & 42,2 & 28,9 & $\begin{array}{l}0,1 \\
8\end{array}$ \\
\hline $\begin{array}{l}\text { Problemas com o } \\
\text { Sono }\end{array}$ & 33,3 & 37,1 & 1 & 22,2 & 0 & 0 & 28,9 & 22,2 & 0,5 \\
\hline $\begin{array}{l}\text { Problemas de } \\
\text { Atenção }\end{array}$ & 11,1 & 11,1 & 1 & 5,6 & 0 & 0 & 8,9 & 6,7 & 1 \\
\hline
\end{tabular}




\begin{tabular}{|c|c|c|c|c|c|c|c|c|c|}
\hline $\begin{array}{l}\text { Comportamento } \\
\text { Agressivo }\end{array}$ & 37,1 & 29,7 & 0,5 & 33,3 & 5,6 & $\begin{array}{l}0,6 \\
3\end{array}$ & 35,6 & 20 & $\begin{array}{l}0,0 \\
1\end{array}$ \\
\hline $\begin{array}{ll}\text { Escala } & \text { de } \\
\text { Internalização } & \end{array}$ & 44,4 & 37,1 & $\begin{array}{l}0,6 \\
8\end{array}$ & 66,7 & 44,4 & $\begin{array}{l}0,1 \\
2\end{array}$ & 53,3 & 40 & 0,1 \\
\hline $\begin{array}{l}\text { Escala de } \\
\text { Externalização }\end{array}$ & 44,4 & 37,1 & $\begin{array}{l}0,2 \\
1\end{array}$ & 61,1 & 44,4 & $\begin{array}{l}0,0 \\
4\end{array}$ & 51,1 & 40 & $\begin{array}{l}0,1 \\
8\end{array}$ \\
\hline $\begin{array}{l}\text { Escala Total de } \\
\text { Problemas }\end{array}$ & 51,9 & 33,3 & 1 & 77,8 & 50 & $\begin{array}{l}0,0 \\
6\end{array}$ & 62,2 & 40 & 0 \\
\hline \multicolumn{10}{|c|}{ Escalas orientadas pelo DSM } \\
\hline $\begin{array}{l}\text { Problemas } \\
\text { Afetivos }\end{array}$ & 33,3 & 29,7 & $\begin{array}{l}0,1 \\
4\end{array}$ & 38,9 & 22,2 & $\begin{array}{l}0,3 \\
7\end{array}$ & 35,6 & 26,7 & $\begin{array}{l}0,2 \\
8\end{array}$ \\
\hline $\begin{array}{l}\text { Problemas de } \\
\text { Ansiedade }\end{array}$ & 51,9 & 59,2 & 0 & 50 & 38,9 & $\begin{array}{l}0,7 \\
2\end{array}$ & 51,1 & 51,1 & 1 \\
\hline $\begin{array}{l}\text { Problemas } \\
\text { Desenvolvimental }\end{array}$ & 22,2 & 22,2 & $\begin{array}{l}0,6 \\
8\end{array}$ & 61,1 & 22,2 & $\begin{array}{l}0,0 \\
3\end{array}$ & 37,8 & 22,2 & $\begin{array}{l}0,0 \\
9\end{array}$ \\
\hline $\begin{array}{l}\text { Problemas de } \\
\text { Déficit de Atenção } \\
\text { e Hiperatividade }\end{array}$ & 29,7 & 25,9 & $\begin{array}{l}0,2 \\
8\end{array}$ & 38,9 & 22,2 & $\begin{array}{l}0,2 \\
5\end{array}$ & 33,3 & 24,4 & $\begin{array}{l}0,2 \\
1\end{array}$ \\
\hline $\begin{array}{l}\text { Problemas de } \\
\text { Oposição } \\
\text { Desafio }\end{array}$ & 40,8 & 25,9 & $\begin{array}{l}0,1 \\
2\end{array}$ & 38,9 & 16,7 & $\begin{array}{l}0,2 \\
1\end{array}$ & 40 & 22,2 & $\begin{array}{l}0,0 \\
2\end{array}$ \\
\hline
\end{tabular}

$\mathrm{Na}$ análise estatística, comparando todas as crianças com os resultados da CBCL, antes e após o programa de natação, três síndromes apresentaram mudanças significativas após o programa. Sendo elas, comportamento agressivo, total de problemas, e comportamento opositivo desafiador, no total de crianças. Porém, quando separado por gênero, houve uma diferença significativa na melhora dos meninos, apenas em problemas de ansiedade, na síndrome orientada pelo DSM, diferente do resultado da mesma síndrome, segundo o inventario CBCL. Já as meninas tiveram melhoras significativas em quatro síndromes: problemas com o sono, problemas de atenção, problemas externalizantes e problemas de desenvolvimento.

Disponível em: https://www.nucleodoconhecimento.com.br/educacao-fisica/avaliacao- 
Portanto, nota-se que houve melhora significativa no comportamento das crianças em geral e, observando por gênero, as meninas demostraram uma melhora mais significativa em comparação com os meninos.

$\mathrm{Na}$ análise descritiva, quando comparados os momentos antes e após a realização do programa de aulas de natação, as crianças do estudo apresentaram mudança positiva em seu comportamento, houve redução nos casos clínicos em relação as síndromes abordadas pela escala, conforme Tabela 2 .

Tabela 2 Crianças que deixaram de apresentar escores clínicos na CBCL, após o programa de natação.

\begin{tabular}{|c|c|c|c|}
\hline DEIXARAM DE SER CLÍNICOS & Total & Meninos & Meninas \\
\hline Emocional Reativo & 4 & 3 & 1 \\
\hline Ansiedade/Depressão & 3 & 1 & 2 \\
\hline Queixas Somática & 2 & 1 & 1 \\
\hline Isolamento & 6 & 3 & 3 \\
\hline Problemas com Sono & 0 & +1 & 4 \\
\hline Problemas de Atenção & 1 & 0 & 1 \\
\hline Comportamento Agressivo & 7 & 2 & 5 \\
\hline Problemas Internalizantes & 6 & 2 & 4 \\
\hline Problemas Externalizantes & 5 & 2 & 3 \\
\hline Total de Problemas & 10 & 5 & 5 \\
\hline \multicolumn{4}{|l|}{ Escalas Orientadas pelo DSM } \\
\hline Problemas Afetivos & 4 & 1 & 3 \\
\hline Problemas de Ansiedade & 0 & +2 & 2 \\
\hline Problemas Desenvolvimentais & 7 & 0 & 7 \\
\hline Problemas Desenvolvimentais, & 4 & 1 & 3 \\
\hline Problemas de Oposição e Desafio & 8 & 4 & 4 \\
\hline
\end{tabular}

Nota. + = aumentou o $\mathrm{N}$ de clínicos 
Análise das observações dos comportamentos das crianças feita pela professora/pesquisadora ao longo do programa, figura 1.

FIGURA 1 Os valores da análise feita durante o programa para cada comportamento observado pela professora.

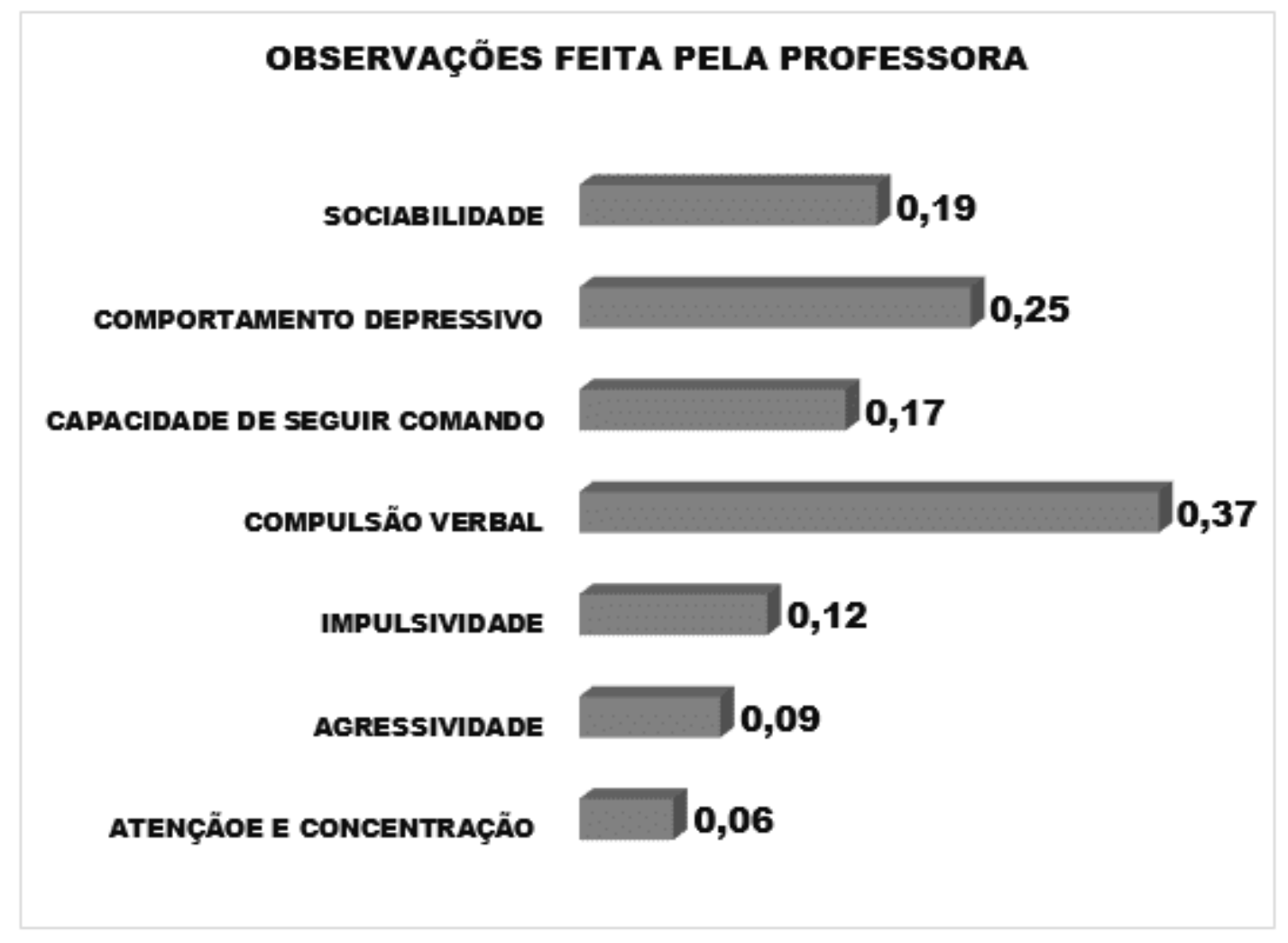

Na comparação da primeira aula com a última, não houve melhora significativa no comportamento das crianças em geral. Em contrapartida, na percepção da pesquisadora, no contato diário com as crianças, e também de acordo com relato (feedback) dos pais e/ou responsáveis, houve melhora significativa no comportamento geral da maioria das crianças.

Essas melhoras foram constatadas em atitudes exibidas durante as aulas, como a diminuição da ansiedade, inclusive de um participante com diagnóstico médico e prescrição de medicação, melhora da atenção, melhora da disciplina, melhora da agressividade, melhora na capacidade de seguir comando, no respeito aos colegas, 
à professora e às mães/cuidadoras. Observou-se também que um menino teve piora no seu comportamento, tornando-se mais agressivo, teimoso, sem entusiasmo para as atividades e menos ainda para recreações, saindo da aula sem avisar. Essa piora, de acordo com o feedback da mãe, pode ter sido decorrente de fatores externos, como a gravidez da mãe de um terceiro filho, o que tornaria o participante o filho do meio, além disso, a professora da escola regular também estava gravida. Tais fatos podem indiciar que o menino se sentia mais inseguro e/ou com medo de ter menos atenção dos pais/familiares e também da professora da escola.

\section{DISCUSSÃO}

Participaram do estudo 45 crianças de 3 a 5 anos, sendo 27 meninos (60\%) e 18 meninas (40\%), com predominância da etnia branca, com 43 (95,6\%) e 2 pardos $(4,4 \%)$, os 45 pais mantinham união estável no período do estudo. Quanto à escolaridade e a situação econômica, os pais tinham nível cultural de médio a alto. Assim, as características observadas na amostra evidenciaram uma população homogênea, apresentando o mesmo nível socioeconômico.

A avaliação comportamental de crianças pré-escolares contribuiu para identificar alterações importantes na melhora do comportamento após o programa de natação. Essa avaliação detectou melhora considerável na diminuição dos casos clínicos após o programa. Tendo em vista a escassez de estudos com crianças nessa faixa etária, existe uma dificuldade em encontrar uma quantidade significativa de resultados semelhantes. Borsa, Souza \& Bandeira (2011); Moura, Marinho-Casanova, Meurer \& Campana (2008) também tiveram dificuldade de encontrar significância em seus resultados, ao apontarem os problemas externalizantes como os mais frequentes, já que os próprios Borsa, Souza \& Bandeira (2011) indicam que os internalizantes são os mais prevalentes. A saber, neste estudo, em relação aos problemas externalizantes, detectou-se menor redução de casos clínicos, após o programa, enquanto que, em relação aos problemas internalizantes, observou-se maior redução de casos clínicos, e uma redução ainda maior foi observada em relação ao total de problemas. 
Ainda corroborando o fenômeno da subjetividade dos resultados de investigações do comportamento infantil, alguns estudos revelam que os meninos são os que mais apresentam problemas de comportamento, sobretudo os externalizantes, enquanto que as meninas apresentam menos problemas em geral, sendo as queixas em relação a elas referentes aos comportamentos internalizantes (BORSA, SOUZA \& BANDEIRA 2011; BORSA \& NUNES 2011). Tais descobertas diferem dos resultados deste estudo, pois foi observado que dentre os meninos os problemas internalizantes foram mais frequentes, enquanto que os externalizantes das meninas foram mais significativos, essa diferença pode estar relacionada às idiossincrasias dos sujeitos da amostra, além dos fatores associados a diferença de gênero, como características hormonais, conservas culturais e expectativas em relação aos papéis de gênero (BORSA \& COLS., 2011; BORSA E NUNES, 2011).

Autores também identificam que, independentemente da cultura e do espaço geográfico, o desenvolvimento infantil é marcado por transformações comportamentais consideradas importantes, por permitirem à criança a aprendizagem de novas habilidades, e por caracterizarem os comportamentos infantis como desejáveis ou indesejáveis (BOLSONI-SILVA, MARTURANO, \& MANFRINATO, 2005).

Comportamentos desejáveis ou adequados são aqueles resultantes de mudanças que ocorrem como consequência da interação do organismo com o ambiente, permitindo à criança 0 contato com situações importantes de aprendizagem que promovem o desenvolvimento. Os comportamentos indesejáveis, por sua vez, referem-se aos problemas de atitudes decorrentes do excesso ou da falta de situações, e de ambientes que facilitariam e ampliariam as possibilidades de aprendizagem e, em consequência, de desenvolvimento. Os comportamentos indesejáveis manifestam-se em problemas internalizantes ou externalizantes (ACHENBACH, 1991; BOLSONISILVA et al., 2005). Em relação a este quesito, no decorrer do programa aqui proposto, as crianças mostraram melhora gradativa e considerável nos comportamentos desejáveis de acordo com (BOLSONI-SILVA, MARTURANO, \& MANFRINATO, 2005). 
Tendo em vista a vasta gama de variáveis, fica clara a determinação de problemas múltiplos de comportamento e, mesmo sendo, por exemplo, as práticas parentais negativas preditivas de problemas comportamentais, não são essas as variáveis indicadoras exclusivas, embora sejam muito importantes e claramente documentadas (BOLSONI-SILVA, LOUREIRO \& MATURANO, 2011).

Theunissen, et al. (2015) também postulam que crianças com problemas psicológicos são propensas a experienciar dificuldades em vários aspectos do seu funcionamento diário. Estes problemas podem ser graves e persistir por muito tempo. Porém, tendo em vista que um dos critérios de exclusão deste estudo foi diagnostico de doença mental, as crianças participantes deste estudo não apresentaram dificuldades especificas em nenhum aspecto de seu comportamento no programa.

\section{CONCLUSÃO}

Conclui-se que o programa de natação favoreceu na redução dos casos clínico e no comportamento das crianças de 3 a 5 anos. Apesar dos resultados serem uma importante contribuição para os modelos teóricos da avaliação comportamental, é importante considerar as limitações do estudo que estão relacionadas ao viés de amostra, sendo que esta, neste estudo, foi definida pela conveniência, não atendendo aos rigores de aleatoriedade. Outra limitação que precisa ser considerada é o fato de não ter havido grupo controle, contudo, não podendo atribuir a melhora comportamental inteiramente ao programa de natação. Além disso, o tamanho reduzido da amostra e o desenvolvimento e evolução naturais das crianças e o contato direto da pesquisadora com os participantes podem ter dificultado a clareza dos resultados.

Apesar disso, houve melhora no comportamento das crianças, após o programa de natação, observando-se uma melhora considerável, pela análise descritiva, em comparação com a análise estatística nas seguintes síndromes: comportamento agressivo, total de problemas, comportamento opositivo desafiador, problemas com o sono, problemas de atenção, problemas externalizantes e problemas 
desenvolvimentais. De acordo com a análise estatística quantitativa, comparando a primeira aula com a última, não houve melhora significativa no comportamento das crianças em geral. Em contrapartida, na percepção da pesquisadora, no contato diário com as crianças, e também de acordo com relato (feedback) dos pais e/ou responsáveis, houve melhora no comportamento geral da maioria das crianças.

\section{REFERÊNCIAS}

ACHENBACH, T. M. Manual for the Child Behavior Checklist/4-18 and 1991 profile. 1991. Burlington: Departament of Psychiatry, University of Vermont.

ACHENBACH, T. M.; \& RESCORLA, L. A. Manual for the ASEBA preschool forms \& profiles. 2000. Burlington, 05401-3456. Web: www.ASEBA.org.

BENKO, C. R., FARIAS, A. C. \& CORDEIRO, M. L. Eating habits and psychopathology: translation, adaptation, reliability of the nutrition behavior inventory to Portuguese and relation to psychopathology. J Bras Psiquiatr, 2011. 240-6

BOLSONI-SILVA, A. T., MARTURANO, E. M. \& MANFRINATO, J. W. D. Mães avaliam comportamentos socialmente desejados e indesejados de pré-escolares. Psicologia em estudo, Maringá, 10, 2005. 245-252.

BOLSONI-SILVA, A. T., LOUREIRO, S. R. \& MARTURANO, E. D. Problemas de comportamento e habilidades sociais infantis: modalidades de relatos. 42(3), 2011. 354-361.

BORSA, J. C, \& NUNES, M. L. T. Prevalência de problemas de comportamento em uma amostra de crianças em idade escolar da cidade de Porto Alegre. Aletheia, 20011. 34 Canoas abr.

BORSA, J. C., SOUZA, D. S. \& BANDEIRA, D. R. Prevalência dos problemas de comportamento de uma amostra de crianças do Rio Grande do Sul. Psicologia: Teoria e Prática. 13(2):65-29, 2011. 
CIMINO, S., CERnigliA, L., PORRECA, A. SIMONELLI, A., RONCONI, L. \& BALLAROTTO, G. Mothers and Fathers with Binge Eating Disorder and Their 18-36 Months Old Children: A Longitudinal Study on Parent-Infant Interactions and Offspring's Emotional-Behavioral Profiles. Front. Psychol. 7:580, 2016

GRIFFITH UNIVERSITY. "children who swim start smarter, study suggests." ScienceDaily, disponívem em: www.sciencedaily.com/releases/2012/11/121116124603.htm. Acessado em: $16 / 09 / 2015$

JONES-PALM, D. H. \& Palm, J. Physical activity and its impact on health behavior among youth.

Disponível

em: https://www.icsspe.org/sites/default/files/PhysicalActivity.pdf. Acessado em: $12 / 07 / 2015$

KRIEGER, F. V., LEIBENLUFT, STRINGARIS, A. \& POLANCZYK, G. V. Irritability in Children and Adolescents: past concepts, current debates, and future opportunities. Rev. Bras. Psiquiatr. São Paulo: 35 (1), 2013,

LACANNELIER, F., EWERT, J. C. P., GROISSMAN, S., GALLARDO, D.; BARDET, A. M., Bascuñan, A. Validation of child inventory for children ages 1 to 5 years (cbcl 1 5) at the Santiago de Chile city. Univ. Psychol. 13 (2) bogotá apr/june, 2014.

MOURA, C. B., MARINHO-CASANOVA, M. L., MEURER, P. H., \& CAMPANA, C. Caracterização da clientela pré-escolar de uma clínica-escola brasileira a partir do Child Behavior Checklist (CBCL). Contextos Clínicos, 1. (1), 1-8. 2008

PSYCHOGIOU, L.; MOBERLY, N. J.; PARRY, E,; NATH, S.; KALLITSOGLOU, A. \& RUSSELL, G. Parental depressive symptoms, children's emotional and behavioural problems, and parents' expressed emotion-Critical and positive comments. PLoS ONE 12(10) 0183546. 2017 
ROHENKOHL, L. M. I. A., CASTRO, E. K. Afetividade, conflito familiar e problemas de comportamento em pré-escolares de famílias de baixa renda: visão de mães e professoras. Psicol. Cienc. Prof. 32 (2) Brasília.2012

SELKE, L. A. Can Newborn Babies Swim? Livestrong.com. Disponível em http://www.livrestrong.com/article/237480-can-newborn-babies-swim/ Acessado em $13 / 03 / 2014$

THEUNissen, M. H. C., VOGELS, A. G. C., WOlFF, M. S., CRONE, M. R. \& REIJNEVELD, S. A. Comparing three short questionnaires to detect psychosocial problems among 3 to 4-year olds. BMC Pediatrics, Disonível em: at:http://www.biomedcentral.com/1471-2431/15/84. Acessado em: 24/03/2015

WEST, W. Do early Swimming Lessons Give Kids the Best Start? Family Edge Human Dignity \& the Family. 30 november 2012.

Enviado: Maio, 2019.

Aprovado: Outubro, 2019. 